\title{
Multimodal Function Optimizing by a New Hybrid Nonlinear Simplex Search and Particle Swarm Algorithm
}

\author{
Fang Wang and Yuhui Qiu \\ Intelligent Software and Software Engineering Laboratory, \\ Southwest China Normal University, Chongqing, 400715, China \\ \{teresa78, yhqiu\} @swnu.edu.cn
}

\begin{abstract}
A new hybrid Particle Swarm Optimization (PSO) algorithm is proposed in this paper based on the Nonlinear Simplex Search (NSS) method for multimodal function optimizing tasks. At late stage of PSO process, when the most promising regions of solutions are fixed, the algorithm isolates particles that fly very close to the extrema and applies the NSS method to them to enhance local exploitation searching. Explicit experimental results on famous benchmark functions indicate that this approach is reliable and efficient, especially on multimodal function optimizations. It yields better solution qualities and success rates compared to other three published methods.
\end{abstract}

\section{Introduction}

A new research field called Swarm Intelligence (SI) arose [1], [2] in the beginning of 90's, which focuses on analogies of swarm behavior of natural creatures. These optimization techniques suggest that the main ideas of intelligent individuals' sociocognition can be effectively introduced to develop efficient optimization algorithms. Among all SI algorithms, the Ant Colony Optimization (ACO) proposed by Dorigo [3] is the most well known technique and is mainly used for combinatorial optimization tasks. The Particle Swarm Optimization (PSO) is another SI method, which is mostly used for continuous function optimizing and has been originally proposed by R.C. Eberhart and J. Kennedy [4]. PSO exhibits good performance in solving hard optimization problems and engineering applications, and compares favorably to other optimization algorithms [5], [6].

Numerous variations of the basic PSO algorithm have been projected by researchers in this field [7] to improve its overall performance since its introduction in 1995. Hybrid PSO algorithms with determinate methods, such as the Nonlinear Simplex Search method, are proved to be superior to the original two techniques and have many advantages over other techniques, such as Genetic Algorithms (GAs) and Tabu Search (TS), because these hybrid methods can perform exploration search with PSO and exploitation search with determinate methods [8]. Generating initial swarm by the NSS might improve, but is not satisfying for multimodal function optimizing tasks [9]. Developing the NSS as an operator to the swarm during the optimization may increase the computational complex considerably. 
In this paper, the Nonlinear Simplex Search method is adopted at late stage of PSO algorithm when particles fly quite near to the extrema. Experimental results on several famous test functions show that this is a very promising way to increase both the convergence speed and the success rate significantly. We briefly introduce the PSO algorithm and the nonlinear simplex search method in section 2 . In section 3 , the proposed algorithm and experimental design is described, correlative results of experiments are exhibited in section 4 . The paper comes to the end with terse conclusions and some ideas for further work.

\section{Background Knowledge}

\subsection{The Particle Swarm Algorithm}

As mentioned in previous section, the particle swarm algorithm is proposed mainly for continuous optimization tasks based on the analogy of swarm of bird and fish school. In PSO algorithm, the population of potential solutions is called swarm, which is usually initialized by a uniform distribution over the search space and then iteratively explores the search space, simulating the movement of a "birds' flock" while searching for food. During the search process, global exchange of information among all individuals, which are called particles, takes place so that each particle can benefit from the current search results of other particles.

In the original PSO formulae, particle $\mathrm{i}$ is denoted as $\mathrm{X}_{\mathrm{i}}=\left(\mathrm{x}_{\mathrm{i} 1}, \mathrm{x}_{\mathrm{i} 2}, \ldots, \mathrm{x}_{\mathrm{iD}}\right)$, which represents a potential solution to a problem in $D$-dimensional space. Each particle maintains a memory of its previous best position, $P_{i}=\left(p_{i 1}, p_{i 2}, \ldots, p_{i D}\right)$, and a velocity along each dimension, represented as $\mathrm{V}_{\mathrm{i}}=\left(\mathrm{v}_{\mathrm{i} 1}, \mathrm{v}_{\mathrm{i} 2}, \ldots, \mathrm{v}_{\mathrm{iD}}\right)$. At each iteration, the $\mathrm{P}$ vector of the particle with the best fitness in the swarm, designated $\mathrm{g}$, and the $\mathrm{P}$ vector of the current particle are combined to adjust the velocity along each dimension, and that velocity is then used to compute a new position for the particle.

The velocity and position of particle $i$ at $(t+1)$ th iteration are updated by [5]:

$$
\begin{aligned}
& v_{i d}^{t+1}=w^{*} v_{i d}^{t}+c_{1} * r_{1}^{t} *\left(p_{i d}^{t}-x_{i d}^{t}\right)+c_{2} * r_{2}^{t}\left(p_{g d}^{t}-x_{i d}^{t}\right) \\
& x_{i d}^{t+1}=x_{i d}^{t}+v_{i d}^{t+1}
\end{aligned}
$$

Constants $c_{1}$ and $c_{2}$ determine the relative influence of the social and cognition components (learning rates), which often both are set to the same value to give each component equal weight; $r_{1}$ and $r_{2}$ are random numbers uniformly distributed in the interval $[0,1]$. A constant, Vmax, was used to limit the velocities of the particles. The parameter $\mathrm{w}$, which was introduced as an inertia factor, can dynamically adjust the velocity over time, gradually focusing the PSO into a local search [6].

Maurice Clerc has derived a constriction coefficient $\mathrm{K}$, a modification of the PSO that runs without Vmax, reducing some undesirable explosive feedback effects [10], [11]. The constriction factor is computed as:

$$
K=\frac{2}{\left|2-\varphi-\sqrt{\varphi^{2}-4 \varphi}\right|}, \varphi=c_{1}+c_{2}, \varphi>4
$$


With the constriction factor K, the PSO formula for computing the new velocity is:

$$
v_{i d}^{t+1}=k^{*}\left(v_{i d}^{t}+c_{1} * r_{1}^{t} *\left(p_{i d}^{t}-x_{i d}^{t}\right)+c_{2} * r_{2}^{t}\left(p_{g d}^{t}-x_{i d}^{t}\right)\right)
$$

Carlisle and Dozier investigated the influence of different parameters in PSO, selected $\mathrm{c}_{1}=2.8, \mathrm{c}_{2}=1.3$, population size as 30 , and proposed the Canonical PSO [12].

To speed up the convergence process and avoid premature problem, Shi YH [13] proposed a method to linearly decrease the inertia weight (LDW) by:

$$
w=w_{\max }-\left(w_{\max }-w_{\min }\right) * \frac{\text { iter }}{\text { iter }_{\max }}
$$

Where $\mathrm{w}_{\max }$ and $\mathrm{w}_{\min }$ is the maximum and minimum of inertia weight, iter is the current iteration number, iter $_{\max }$ is the maximum iteration times.

\subsection{The Nonlinear Simplex Search Method}

Spendley et al presented the basic simplex method in 1962, which is an efficient sequential optimization method for function minimization tasks, and then improved by Nelder and Mead, to what is called the Nonlinear Simplex Search method [14]. It needs only values and not derivatives of the objective function. In general, the NSS is considered as the best method if the figure of merit is "get something to work quickly".

A $D$-dimensional simplex is a geometrical figure consisting of $\mathrm{D}+1$ vertices $(D$ dimensional points) and all their interconnecting segments, polygonal faces etc. We consider only simplexes that are non-degenerated, i.e., that enclose a finite inner $D$ dimensional volume.

The NSS starts with an initial simplex generated using the found minimum as one of its vertices and generating the rest D points randomly. Then it takes a series of steps as follows to rescale the simplex: first, it finds the points where the objective function is highest (the least favorable trial $\mathrm{W}$ ) and lowest (the most favorable trial $\mathrm{B}$ ); then it reflects the simplex around the high point to point $\mathrm{R}$. If the solution is better, it tries an expansion in that direction to point $\mathrm{E}$; else if the solution is worse than the second-highest (next-to-the worst) point $\mathrm{Nw}$, it tries an intermediate point. When the method reaches a "valley floor", the simplex is contracted in the transverse direction in order to ooze down the valley or it can be contracted in all directions, pulling itself in around its lowest point, and started again.

The different moves of a two-dimensional simplex are shown in Figure 1.

At each step, the rejected trial $\mathrm{W}$ is replaced by one of the following trials on conditions that:

$$
\begin{aligned}
& R=\bar{C}+\alpha(\bar{C}-W), \text { if } f_{B}<f_{R}<f_{N w} \\
& E=\bar{C}+\gamma(\bar{C}-W), \text { if } f_{R}<f_{B} \\
& C+=\bar{C}+\beta^{+}(\bar{C}-W), \text { if } f_{N w}<f_{R}<f_{M} \\
& C-=\bar{C}-\beta^{-}(\bar{C}-W), \text { if } f_{R}>f_{M}
\end{aligned}
$$


Where $\bar{C}$ is the centroid of the remaining vertices; $\alpha, \gamma, \beta^{+}$and $\beta^{-}$is coefficients of reflection, expansion, positive contraction and negative contraction; $f_{B}, f_{N w}, f_{W}$ and $f_{R}$ is the values of object function on point $\mathrm{B}, \mathrm{Nw}, \mathrm{W}$ and $\mathrm{R}$ respectively.

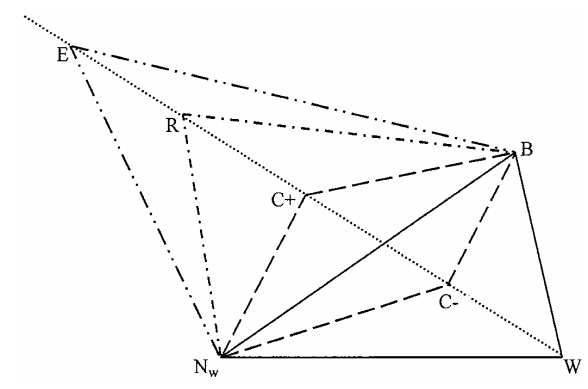

Fig. 1. Different simplex moves from the rejected trial condition, $\mathrm{W}=$ the rejected trial, $\mathrm{R}=$ reflection, $\mathrm{E}=$ expansion, $\mathrm{C}+=$ positive contraction, $\mathrm{C}-\mathrm{=}$ negative contraction.

\section{The Proposed Algorithm and Experimental Design}

At late stage of PSO running, promising regions of solutions have been located. Applying the NSS to enhance exploitation search at this stage is capable of improving the solution quality and convergence rate.

We propose a hybrid Nonlinear Simplex Search PSO (NSSPSO) based on the Canonical PSO algorithm, which isolates a particle and apply the NSS to it when it reaches quite close to the extrema (within the diversion radius). If the particle "lands" within a specified precision of a goal solution (error goal) during the NSS running, a PSO process is considered to be successful, otherwise it may be laid back to the swarm and start the next PSO iteration.

The diversion radius is computed as:

$$
\begin{aligned}
& \text { DRadius }=\text { ErrorGoal }+\delta \\
& \delta=\left\{\begin{array}{c}
100 * \text { ErrorGoal, if ErrorGoal }<=10^{-4} \\
0.01 * \text { ErrorGoal, otherwise }
\end{array}\right.
\end{aligned}
$$

In a NSS process, an initial simplex is consists of the isolated particle $i$ and other D vertices randomly generated with the mean of $X_{i}$ and standard deviation of DRadius.

The stopping criterion is defined as:

$$
\left|f\left(X_{g}\right)-G M\right|<\text { ErrorGoal }
$$

In order to get quicker convergence, we set maximums of iterations in all experiments as a second stopping criterion. In the later case, we consider the search process to be failed. 
The benchmark functions [15] on which the proposed algorithm has been tested and compared to other methods in the literature, and the corresponding parameters are listed in Table 1.

Table 1. Benchmark functions used in our experiments

\begin{tabular}{ccccc}
\hline Function & Xmax & GM & Error Goal & Dimension \\
\hline Sp: Sphere & \multirow{2}{*}{100} & 0 & $10^{-7}$ & 2 \\
& & & $10^{-7}$ & 10 \\
& & & $10^{-4}$ & 30 \\
Ro: Rosenbrock & 30 & 0 & $10^{-7}$ & 2 \\
& & & 10 & 10 \\
& & & 100 & 30 \\
Ra: Rastrigin & 5.12 & 0 & $10^{-7}$ & 2 \\
& & & 10 & 10 \\
& & & 100 & 30 \\
Gr: Griewank & 600 & Dimension-1 & $10^{-7}$ & 2 \\
& & & $10^{-1}$ & 10 \\
Sc: Schaffer & 5.12 & -1 & $10^{-1}$ & 30 \\
L3: Levy No.3 & 10 & -176.5418 & $10^{-4}$ & 2 \\
L8: Levy No.8 & 10 & 0 & $10^{-4}$ & 2 \\
Co: Corana & 5.12 & 0 & $10^{-7}$ & 3 \\
Fr: Freudenstein & 10 & 0 & $10^{-7}$ & 4 \\
Go: Goldstern & 5.12 & 3 & $10^{-7}$ & 2 \\
\hline
\end{tabular}

*Note: for function Sphere, Rosenbrock, Rastrigin and Griewank, three Error Goals are given for separate dimension of three tests. GM is the known theoretic extremum of each test function.

To eliminate the influence of different initial swarms, we implement 200 experiments for each test and the maximum number of PSO iterations is set to be 500, swarm size is 60 for 30-dimension functions and 30 for others. We use symmetric search spaces as [-Xmax...Xmax], and set Vmax=Xmax. In LDW PSO algorithm, $\mathrm{c}_{1}=\mathrm{c}_{2}=2.0$, and the inertia weight $\mathrm{w}$ was initially set to 0.9 and gradually decreased toward 0.4. Parameters used in the NSS are: $\alpha=1.0, \gamma=2.0, \beta^{+}=\beta^{-}=0.5$. We reimplemented all the algorithms mentioned in Section 4 and executed them under the same environment: Matlab 7.0 [16], Pentium IV 2.8GHz CPU, 512M RAM, Windows2000 Professional OS.

\section{Experimental Results}

The rate of success, mean function evaluations, average optima and total CPU time for each test are listed in Table 2 4. The subscripts of test functions denote different 
dimensions. The proposed algorithm is denoted as NSSPSO, NS-PSO is another NSS hybrid PSO proposed by Parsopoulos and Vrahatis [9], LDW represents Linearly Decreasing Weight method by Shi YH [5], [6], and CPSO is the Canonical PSO by Carlisle A [12]. From the tables we can see that the overall performance of NSSPSO algorithm is apparently superior to the other 3 algorithms in terms of success rate, solution quality and convergence speed as well, especially on multimodal functions such as Levy No.3, Schaffer, Rosenbrock and Griewank. As to high dimension function optimizing, NSSPSO operates appreciably inferior to NS-PSO due to its computational expense, but is still equal to the Canonical PSO algorithm in most cases. LDW PSO yields poorest performance in nearly all tests.

Table 2. Rate of success, mean function evaluations for each test function

\begin{tabular}{|c|c|c|c|c|c|c|c|c|}
\hline & \multicolumn{4}{|c|}{ Rate of success } & \multicolumn{4}{|c|}{ Mean function evaluations } \\
\hline & NSSPSO & NS-PSO & CPSO & LDW & NSSPSO & NS-PSO & CPSO & LDW \\
\hline $\mathrm{Ro}_{2}$ & 0.99 & 0.845 & 0.97 & 0.075 & 7589.6 & 9421.8 & 8450.3 & 14876 \\
\hline $\mathrm{Ra}_{2}$ & 1 & 0.84 & 1 & 0.94 & 2970.6 & 5573.3 & 3181.8 & 9627.1 \\
\hline $\mathrm{Gr}_{2}$ & 0.8 & 0.685 & 0.735 & 0.335 & 8383.9 & 9397.6 & 8985.9 & 13752 \\
\hline $\mathrm{Sc}$ & 0.7 & 0.57 & 0.645 & 0.2 & 8662.6 & 9906.8 & 9119.7 & 14194 \\
\hline L3 & 1 & 0.83 & 1 & 0.155 & 2595.7 & 4827 & 2853.3 & 14194 \\
\hline L8 & 1 & 0.995 & 1 & 1 & 2259.9 & 2045 & 2327.6 & 8526.6 \\
\hline Co & 1 & 0.99 & 1 & 1 & 6493.7 & 4877.4 & 5524.5 & 12159 \\
\hline $\mathrm{Fr}$ & 0.98 & 0.52 & 0.975 & 0.05 & 4466.6 & 9321.1 & 4349.6 & 10629 \\
\hline Go & 1 & 0.94 & 1 & 1 & 2844.5 & 3601.2 & 2955.4 & 8510.9 \\
\hline $\mathrm{Sp}_{10}$ & 1 & 1 & 1 & 0.35 & 9677.9 & 5723.4 & 6306.8 & 14948 \\
\hline $\mathrm{Ro}_{10}$ & 0.83 & 0.945 & 0.84 & 0.62 & 5365.1 & 3552 & 5461.6 & 13600 \\
\hline $\mathrm{Ra}_{10}$ & 0.963 & 0.83 & 0.96 & 0.71 & 5390.7 & 5929.5 & 5094.3 & 13471 \\
\hline $\mathrm{Gr}_{10}$ & 0.845 & 0.8 & 0.845 & 0.515 & 8997.5 & 7084.1 & 7332.3 & 14124 \\
\hline $\mathrm{Sp}_{30}$ & 1 & 1 & 1 & 0 & 11716 & 13789 & 15448 & 30060 \\
\hline $\mathrm{Ro}_{30}$ & 0.825 & 0.94 & 0.795 & 0 & 14945 & 10697 & 16576 & 30060 \\
\hline $\mathrm{Ra}_{30}$ & 0.995 & 1 & 1 & 0.295 & 8987.1 & 3475.5 & 8511.3 & 29604 \\
\hline $\mathrm{Gr}_{30}$ & 0.99 & 1 & 0.995 & 0 & 11019 & 8999.1 & 10801 & 30060 \\
\hline
\end{tabular}

\section{Conclusions and Future Work}

In this paper, we propose a new hybrid Particle Swarm Optimization algorithm, which applies the Nonlinear Simplex Search method at late stage of PSO running when the most promising regions of solutions have been located. We implement wide variety of experiments on well-known benchmark functions to test the proposed algorithm. The results compared to other 3 published methods demonstrate that this method is very effective and efficient, especially for continuous multimodal function optimization tasks. 
Table 3. Average optima for each test function

\begin{tabular}{lccccc}
\hline \multirow{2}{*}{$\begin{array}{l}\text { Test } \\
\text { Function }\end{array}$} & \multirow{2}{*}{$\mathrm{GM}$} & \multicolumn{4}{c}{ Average optima } \\
\cline { 3 - 6 } $\mathrm{Ro}_{2}$ & 0 & NSSPSO & NS-PSO & CPSO & LDW \\
$\mathrm{Ra}_{2}$ & 0 & $4.8299 \mathrm{e}-8$ & 0.76162 & 0.002373 & 1.3522 \\
$\mathrm{Gr}_{2}$ & 1 & 1.0012 & 1.003 & 1.0016 & 1.0057 \\
$\mathrm{Sc}$ & -1 & -0.99716 & -0.99334 & -0.99664 & -0.99269 \\
$\mathrm{~L} 3$ & -176.5418 & -176.54 & -163.17 & -176.54 & -175.04 \\
$\mathrm{~L} 8$ & 0 & $5.9397 \mathrm{e}-9$ & $5.8974 \mathrm{e}-9$ & $6.0348 \mathrm{e}-9$ & $6.4366 \mathrm{e}-9$ \\
$\mathrm{Co}$ & 0 & $7.7393 \mathrm{e}-9$ & $5.0382 \mathrm{e}-6$ & $6.7979 \mathrm{e}-9$ & $6.67 \mathrm{e} 6 \mathrm{e}-9$ \\
$\mathrm{Fr}$ & 0 & 0.97969 & 80.237 & 1.2246 & 7.3476 \\
$\mathrm{Go}$ & 3 & 3 & 104.1 & 3 & 3 \\
$\mathrm{Sp}$ & 0 & $8.9723 \mathrm{e}-9$ & $8.2146 \mathrm{e}-9$ & $8.2073 \mathrm{e}-9$ & $1.0912 \mathrm{e}-7$ \\
$\mathrm{Ro}_{10}$ & 0 & 298.01 & 461.95 & 20.688 & 372.75 \\
$\mathrm{Ra}_{10}$ & 0 & 9.6195 & 10.14 & 9.7158 & 10.585 \\
$\mathrm{Gr}_{10}$ & 9 & 9.0979 & 9.1038 & 9.1003 & 9.1272 \\
$\mathrm{Sp}_{30}$ & 0 & $9.2039 \mathrm{e}-5$ & $9.3474 \mathrm{e}-5$ & $9.2407 \mathrm{e}-5$ & 47.088 \\
$\mathrm{Ro}_{30}$ & 0 & 2009.3 & 100.63 & 2856.7 & 79609 \\
$\mathrm{Ra}_{30}$ & 0 & 97.418 & 95.818 & 97.752 & 122.48 \\
$\mathrm{Gr}_{30}$ & 29 & 29.098 & 29.094 & 29.094 & 30.487 \\
\hline & & & & & \\
\hline
\end{tabular}

Table 4. Total CPU time for each test function

\begin{tabular}{lccccc}
\hline \multirow{2}{*}{$\begin{array}{l}\text { Fest } \\
\text { Function }\end{array}$} & \multicolumn{5}{c}{ Total CPU time } \\
\cline { 2 - 6 } & NSSPSO & NS-PSO & CPSO & LDW & Improved \\
\hline $\mathrm{Ro}_{2}$ & 31.031 & 36.422 & 31.781 & 59.984 & $2.4 \sim 48.3 \%$ \\
$\mathrm{Ra}_{2}$ & 14.875 & 27.156 & 14.906 & 48.156 & $0.2 \sim 69.1 \%$ \\
$\mathrm{Gr}_{2}$ & 42.469 & 49.141 & 44.813 & 72.656 & $5.2 \sim 41.5 \%$ \\
$\mathrm{Sc}$ & 30.609 & 35.484 & 31.047 & 52.031 & $1.4 \sim 74.5 \%$ \\
$\mathrm{~L} 3$ & 13.063 & 22.688 & 12.813 & 69.578 & $-1.9 \sim 81.2 \%$ \\
$\mathrm{~L} 8$ & 11.953 & 10.891 & 11.188 & 43.234 & $-9.7 \sim 72.3 \%$ \\
$\mathrm{Co}$ & 36.719 & 26.219 & 28.75 & 65.922 & $-29.6 \sim 44.3 \%$ \\
$\mathrm{Fr}_{\mathrm{Go}}$ & 16.672 & 31.875 & 14.516 & 38.094 & $-4.8 \sim 56.2 \%$ \\
$\mathrm{Sp}_{10}$ & 10.5 & 12.859 & 9.8125 & 30.438 & $-7.0 \sim 65.6 \%$ \\
$\mathrm{Ro}_{10}$ & 40.453 & 23.125 & 24.656 & 65.016 & $-34.9 \sim 39.8 \%$ \\
$\mathrm{Ra}_{10}$ & 22.172 & 15.266 & 22.547 & 60.672 & $-45.2 \sim 63.5 \%$ \\
$\mathrm{Gr}_{10}$ & 29.359 & 32.625 & 27.641 & 78.469 & $-6.2 \sim 62.6 \%$ \\
$\mathrm{Sp}_{30}$ & 102.311 & 42.25 & 43.156 & 89.313 & $-102.1 \sim-14.5 \%$ \\
$\mathrm{Ro}_{30}$ & 241.98 & 67.594 & 68.609 & 151.52 & $-187.9 \sim-59.7 \%$ \\
$\mathrm{Ra}_{30}$ & 79.952 & 56.281 & 80.047 & 162.58 & $-22.6 \sim 50.8 \%$ \\
$\mathrm{Gr}_{30}$ & 64.094 & 47.574 & 60.75 & 323.47 & $-19.7 \sim 80.2 \%$ \\
\hline & 83.063 & 71.609 & 81.547 & 269.13 & $-11.9 \sim 69.1 \%$ \\
\hline
\end{tabular}


Future work may focus on accelerating the convergence for high dimension problems, extending the approach to constrained multi-objective optimization, and applying the proposed technique in real hybrid intelligent systems.

\section{References}

1. Bonabeau, E., Dorigo, M., Theraulaz, G.: Swarm Intelligence: From Natural to Artificial Systems, Oxford Press (1999)

2. Kennedy, J., Eberhart, R. C.: Swarm Intelligence, Kaufmann Publishers, Morgan (2001)

3. Dorigo, M.: Optimization, Learning and Natural Algorithms. Ph.D.Thesis, Politecnico di Milano, Italy (1992) (in Italian)

4. Kennedy, J., Eberhart, R. C.: Particle swarm optimization. Proceedings of IEEE International Conference on Neural Networks, Piscataway, NJ (1995) 1942-1948

5. Shi, Y. H., Eberhart, R. C.: A modified particle swarm optimizer. Proceedings of the IEEE Congress on Evolutionary Computation (CEC 1998), Piscataway, NJ (1998) 69-73

6. Shi, Y. H., Eberhart, R. C.: Parameter selection in particle swarm optimization. Evolutionary Programming VII: Proceedings of the Seventh Annual Conference on Evolutionary Programming, New York (1998) 591-600

7. Parsopoulos, K. E., Vrahatis, M. N.: Recent approaches to global optimization problems through particle swarm optimization, Natural Computing, Vol. 1 (2002) 235-306

8. Shu-Kai S. Fan, Yun-Chia Liang, Erwie Zahara: Hybrid Simplex Search and Particle Swarm Optimization for the Global Optimization of Multimodal Functions, Engineering Optimization, Vol. 36, No. 4 (2004) 401-418

9. Parsopoulos, K. E., Vrahatis, M. N.: Initializing the particle swarm optimizer using the nonlinear simplex Method, in Grmela, A. and Mastorakis, N. E. (eds.) Advances in Intelligent Systems, Fuzzy Systems, Evolutionary Computation, WSEAS Press (2002) 216-221

10. Clerc, M.: The swarm and the queen: towards a deterministic and adaptive particle swarm optimization. Proceedings of the IEEE Congress on Evolutionary Computation (1999) 1951-1957

11. Clerc, M., Kennedy, J.: The particle swarm-explosion, stability, and convergence in a multidimensional complex space, IEEE Transactions on Evolutionary Computation, Vol. 6, No. 1, (2002) 58-73

12. Carlisle, A., Dozier, G.: An off-the-shelf PSO. Proceedings of the Workshop on Particle Swarm Optimization, Indianapolis (2001)

13. Shi, Y. H., Eberhart, R. C.: Empirical study of particle swarm optimization. Proceedings of the IEEE Congress on Evolutionary Computation (CEC 1999), Piscataway, NJ (1999) 1945-1950

14. Nelder, J., Mead, R.: A simplex method for function minimization. Computer Journal, Vol. 7 (1965) 308-313

15. Levy, A., Montalvo, A., Gomez, S., et al.: Topics in Global Optimization, SpringerVerlag, New York (1981)

16. Birge, B.: PSOt: a particle swarm optimization toolbox for use with MATLAB. Proceedings of the IEEE Swarm Intelligence Symposium 2003 (SIS 2003), Indianapolis, Indiana, USA (2003) 182-186 